\title{
A warm and friendly memorial session for Helmut Oeschler
}

\author{
Jean Cleymans ${ }^{1, \star}$, Boris Hippolyte ${ }^{2}$, Alexander Kalweit ${ }^{3}$, Christian Müntz ${ }^{4}$, and Joachim Stroth ${ }^{4,5}$ \\ ${ }^{1}$ Department of Physics, University of Cape Town, ZA-7701 Rondebosch, South Africa \\ ${ }^{2}$ Institut Pluridisciplinaire Hubert Curien, Institut d'Études Avancées de l'Université de Strasbourg, France \\ ${ }^{3}$ European Organization for Nuclear Research (CERN), CH-1211 Geneva 23, Switzerland \\ ${ }^{4}$ Institut für Kernphysik (IKF), Goethe-Universität, Max-von-Laue-Str. 1, D-60438 Frankfurt, Germany \\ ${ }^{5}$ GSI Helmholtzzentrum für Schwerionenforschung, Planckstr. 1, D-64921 Darmstadt, Germany
}

\begin{abstract}
A full session was organized in memory of Helmut Oeschler during the 2017 edition of the Strangeness in Quark Matter Conference. It was heart-warming to discuss with the audience his main achievements and share anecdotes about this exceptionally praised and appreciated colleague, who was also a great friend for many at the conference. A brief summary of the session is provided with these proceedings.
\end{abstract}

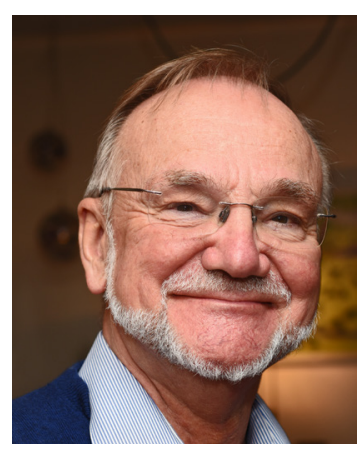

\section{Introduction}

Trying to summarise the studies performed by Helmut Oeschler during his long and fruitful career, a couple of months only after he passed away, was a difficult and emotional task. During a dedicated memorial session at the Strangeness in Quark Matter edition of 2017, four of his closest colleagues and friends presented selected studies, moments and anecdotes, illustrating his successful career and how cheerful and enthusiastic Helmut was for many of us. We hope that, with these proceedings, we manage to convey how positive his presence was among us and how profound and influential his ideas were.

\footnotetext{
^e-mail: Jean.Cleymans@uct.ac.za
} 


\section{Studies in Saclay with SATURNE}

Already in the early eighties Helmut worked on studying reactions of ions at relativistic energies. The goal of Helmut's research was to measure the energy and momentum transfer in reactions of light ions on heavy ones at energies up to $1 \mathrm{AGeV}$. Truly large detector system and respective collaborations did not exist yet. The method applied by him and his colleagues was as clever as it was simple in terms of the instrumentation needed (see Fig. 1). When a fissile nucleus is hit by a relativistic projectile energy and momentum is transferred through knock-out of target nucleons or through stopping of projectile nucleons. In case the damage to the fissile nucleus is moderate, the imparted energy is equilibrated and a compound nucleus is formed. As a consequence, the target nucleus will fission and the two fragments will separate back-to-back with a relative velocity essentially determined by the saddle point configuration. Yet, in the laboratory frame the two fragments are boosted due the momentum transfer to the target nucleus. By measuring the laboratory emission angle and the velocities of the two fragments, the momentum transfer can be reconstructed. Moreover, due to the strong change of the fragment mass distribution for increasing excitation energy (or temperature) of the compound system, or angular distortions due to post-scission particle evaporation from the fission fragments, also the excitation energy could be accessed in the experiment. A series of runs were conducted

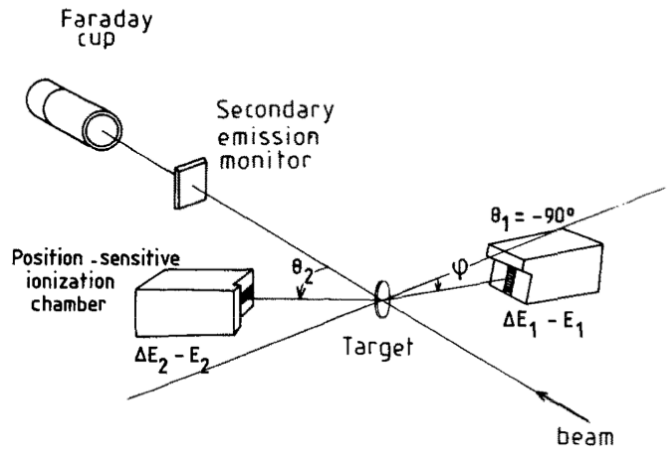

Figure 1. Experimental set-up used to measure linear momentum transfer at SATURNE. The beam hits a target foils tilted by $45^{\circ}$ degrees w.r.t. the beam direction to minimize energy loss in the target. Fission fragments are detected in the ionization chambers, one movable in the reaction plane, the other one out-ofplane. Luminosity was monitored by a Faraday cup. Taken from [1].

at the SATURNE proton synchrotron operated at Saclay, France [1]. Beams of protons, deuterium and helium where used to bombard a Thorium $\left({ }^{232} \mathrm{Th}\right)$ target. The fission fragments where detected by two position sensitive ionization chambers providing total energy and energy-loss information. The position resolution was provided in one dimension only. Therefore, one detector was rotated with respect to the other to allow measuring out-of-plane angles as well. Moreover, the ionization chambers where mounted on moving supports to change the global angular range.

One of the derived observables, the correlation angle $\Theta_{\text {corr }}=\Theta_{2}-\Theta_{1}$, is displayed in Fig. 2. This correlation angle amounts to $180^{\circ}$ if zero momentum is transferred. The three panels show results for three different projectiles $(p, d, \alpha)$ and various beam energies. At first sight, no clear trend is observable. It is important to note, however, that (a) centrality of the collision is not accessible and (b) the method is sensitive only to reactions which result in binary fission. Indeed, the distributions for a proton beam does not change very much except for a growth in width and an emerging asymmetry towards smaller correlation angles. The small vertical arrow at each spectrum indicates the correlation angle if full stopping is achieved. Here a clear trend is visible: full stopping gets less likely as the beam energy increases. The change in reaction mechanism from fusion-fission, the dominant process below the Fermi energy regime, to nucleon knock-out is best visible for argon projectiles. At the lowest energy of $17 \mathrm{AGeV}$, a clear maximum at full transfer is visible with the suppressed shoulder 

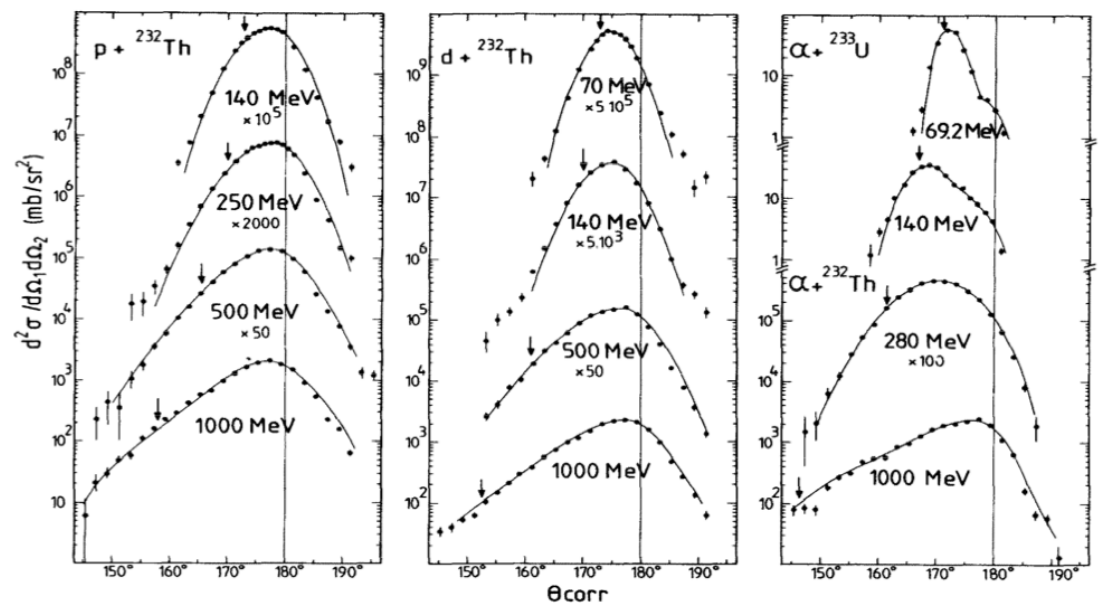

Figure 2. Distribution of measured correlation angles for three different collision systems and different kinetic energies of the projectiles. The lines are drawn to guide the eyes. The vertical lines indicates $\Theta=180^{\circ}$, the case if zero momentum is transferred. Small vertical arrows each mark the correlation angle expected at full stopping.

at larger correlation angles resulting from peripheral transfer reactions. While the energy rises, the maximum of the distributions move to smaller correlation angles as expected for this reaction class but then separates from full momentum transfer and moves back to small transfer.

Helmut was always very much interested in developing his own understanding of the underlying physical principles. In the case of the linear momentum transfer studies, he launched a project in his group at TU Darmstadt to simulate such reaction with a microscopic transport code [2]. At that time available was the Intra Nuclear Cascade Code (INC) and the code of Cugnon. Respective studies revealed that linear momentum transfer primarily results from stopping of projectile nucleons while excitation energy was by large driven by knockout of deeply bound nucleons. Excitation energy finally also defined the limitation of the method (binary fission) but at the same time the discovery of a particular decay of the struck nucleus later called multi fragmentation. This decay channel wheeled substantial cross section to reactions with final states with showing more than two decay products. Consequently, Helmut and his collaborators had to use more and more complex set-ups. On the other hand, Helmut proposed experiments at other laboratories to have heavier beams (GANIL) [3] or higher energies (JINR) [4], setting up such experiments for a small group turned out to be at the edge of what one would consider feasible. Yet he loved these experiments and he continued measurements until the early nineties when already SIS18 and the KaoS experiment (see below) needed most of his attention. Actually, the last measurements of this type he conducted at the Synchrophasotron with Helium beam in 1991, exactly the time, when Russia experienced a historic change with the "Agreement on the Establishment of the Commonwealth of Independent States" signed in Viskuli, Belarus.

Among the many photographs which Helmut took while visiting the laboratories, in particular rousing are those showing him walking around the White House in Moscow in the Fall of 1991. Helmut liked taking photographs a lot. When remembering the great times with Helmut, two attributes come to mind which should not be missed, among many: Helmut took most attention to involve young students in his scientific activities. He enjoyed a lot taking them to the experiments and teaching them not only all the important aspects about doing experiments and the physics addressed. He also gave 
his students the opportunity to share with him all the coming from the cultural aspects of working together with colleagues from other countries and in laboratories there. If one says that science can help bringing nations together, Helmut is a prime example of a colleague who was living this with all his grandeur. Two students of the Technical University of Darmstadt (then TH Darmstadt) graduated with the topic discussed here with doctoral degree. Namely, Gerold Klotz-Engmann covering the earlier experiments and Volker Lips who was analysing data they had taken in Dubna. The authors would like to thank both of them for providing photographs, memories and anecdotes. Like the one about the first student, who was attracted by Helmut to conduct experiments at Saclay. Unfortunately, it never came to it since this student did not really obey the rules for entering the Saclay campus while coming back from the evening excursion in Paris. Officials decided that he had entered Saclay for the last time.

\section{Studies at GSI within the KaoS Collaboration}

Helmut Oeschler joined the KaoS collaboration in the mid 80s. Back in these days, he was still strongly involved in experiments on multi-fragmentation and he was managing Prof. Dr. E. Kankeleit's group at GSI Darmstadt, working on electron and positron production in heavy ion collisions at the Coulomb barrier. Helmut has started his contribution to KaoS by taking responsibility for the particle tracking, in close collaboration with the GSI detector laboratory, where large-area multi-wire proportional counters were built. They were equipped with dedicated readout electronics, developed by the electronics workshop at the nuclear physics department of Technische Hochschule Darmstadt, Helmut's home institute. At that time, the physics agenda of relativistic heavy ion collisions in the $1-\mathrm{AGeV}$ projectile energy regime was mainly driven by questions related to the equation of state of hot and dense nuclear matter. In the early 90 s KaoS started taking data. Helmut's curiosity was excited by the non-monotonic shape of charged pion spectra first reported by the LBL streamer chamber. Hence, he devoted himself and his students to a systematic study of pions produced in heavy ion collisions. This episode was characterized by measuring apparent temperatures of pion spectra, by the emission dynamics of pions during the course of the reaction and by investigating the relevance of exciting and de-exciting of broad baryonic resonances. In this context, Helmut made first contact with the statistical model with the emphasis of compiling a more global picture of particle production in heavy ion collisions. Consequently, at the end of the 90's Helmut's interest shifted towards the KaoS core program, the production of charged kaons and together with his group he made significant contributions on the incompressibility of nuclear matter, as well as emission times and patterns of charged kaons. During that time span with KaoS, Helmut has supervised eight Diploma and five $\mathrm{PhD}$ theses, needless to say, accompanied by numerous conference contributions and publications in renowned journals. Both, the urge to continuously document scientific progress and at the same time mentoring his students with his distinct openhearted and cheerful nature is Helmut's signature feature, which his students and colleagues will always remember.

\section{Studies in Cape Town}

In 1998 Helmut Oeschler spent several weeks at the University of Cape Town. This was the start of an incredibly fruitful scientific collaboration with physicists from this University, resulting in over 30 publications with an average of 82 citations per paper and about 2000 citations. The introduction to one of his papers, written over ten years ago, is still relevant today:

"One of the most remarkable results to emerge from heavy-ion collisions over the past two decades is the striking regularity shown by particle yields at all energies. This has led to several very successful 
proposals describing particle yields over a very wide range of beam energies, reaching from $1 \mathrm{~A} \mathrm{GeV}$

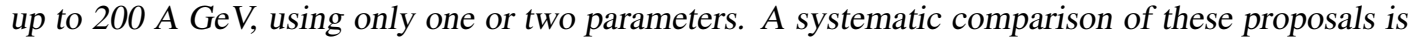
presented here. The conditions of fixed energy per particle, baryon+anti-baryon density, normalized entropy density as well as percolation model are investigated. The results are compared with the most recent chemical freeze-out parameters obtained in the thermal-statistical analysis of particle yields. The sensitivity and dependence of the results on parameters is analyzed and discussed. It is shown that in the energy range above the top AGS energy, within present accuracies, all chemical freeze-out criteria give a fairly good description of the particle yields." [5]

His first visit to South Africa was followed by many subsequent ones, several times with his family.

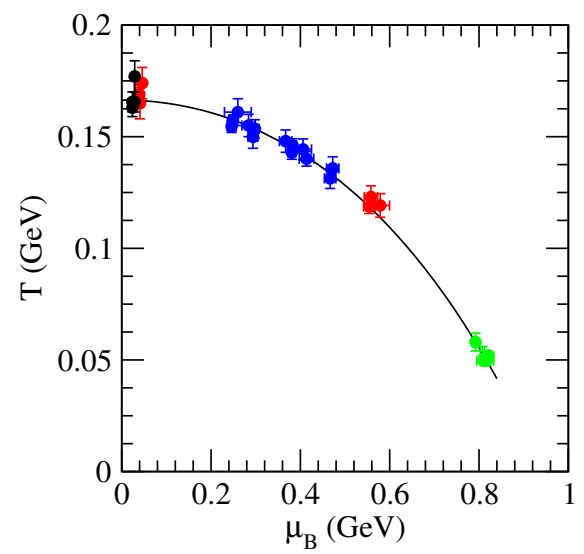

Figure 3. Values of $\mu_{B}$ and $T$ for different energies. The solid line is a parameterization obtained from fits to data. Taken from [5].

Helmut went way beyond the writing of papers and was involved at a personal level in helping the South African community of physicists to be part of the ALICE Collaboration at CERN as expressed by Zinhle Buthelezi: Still can't believe about Helmut! He was a good friend and ally of South Africa. He helped us tremendously to navigate CERN and the ALICE collaborations when we joined. He will be sorely missed!

In South Africa we remember Helmut Oeschler as a person full of enthusiasm, full of energy, with new ideas, trying to understand physics at an intuitive level, never happy with complicated explanations. So full of life that it is hard comprehend what happened so suddenly.

\section{Studies at CERN within the ALICE Collaboration}

In 2000, Helmut joined the ALICE Collaboration as team leader of the Technical University Darmstadt. From the beginning, he was one of the leading scientists in the study of light flavour hadrons at LHC energies. He prepared an important part of the ALICE Collaboration's physics programme associated with the production of these hadrons and anticipate their thermal analysis [6]. He was also one of the key contributors for topics related to particle identification using the central detectors and lead the efforts for the preparation of the publication on identified hadrons (illustrated by Fig. 4) for the very first proton-proton collisions recorded at the LHC at the center of mass energy of $0.9 \mathrm{TeV}$ [7]. Among many roles he had in the collaboration, he served as chair of the ALICE Editorial Board for several years. As of today, the ALICE Collaboration has more than 1800 members from more than 90 countries and produced more than 180 publications for many of which Helmut was instrumental with shaping and organising the scientific content. Helmut truly enjoyed the very international and intercultural environment, being a mentor not only for his own students and post-docs, but for many young 


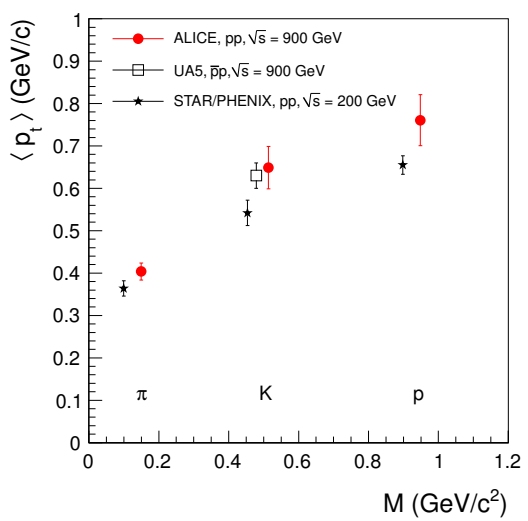

Figure 4. Mean $p_{t}$ as a function of the mass of the emitted hadron in proton-proton collisions at $\sqrt{s}=900 \mathrm{GeV}$ with ALICE at the LHC (red solid circles) and compared to results at $200 \mathrm{GeV}$ obtained at RHIC. Taken from [7].

scientists from all over the world. His scientific interests covered a broad range of topics, but his main focus was on the production of strangeness from low to high energies as well as from small to large colliding systems. Helmut strongly believed in the applicability of the thermal-statistical approach to high multiplicity proton-proton and proton- $\mathrm{Pb}$ collisions and thus it was extraordinary for him to witness the observation of strangeness enhancement in these collision systems by ALICE. In addition, he was fascinated by the complex study of anti-nuclei and hadronic resonances. In particular, Helmut advanced our understanding of the production of the $\phi$-meson and the description of light anti- and hyper-nuclei formation via coalescence and with thermal-statistical models.

For many years, Helmut served as a member of the International Advisory Committee of Strangeness in Quark Matter Conference. This was his favourite conference and he always played a pivotal role in the preparation and presentation of the ALICE results. We will certainly follow his path in addressing some of the most important unresolved problems in this field.

\section{Acknowledgements}

The authors are extremely grateful to the SQM 2017 Organising Committee who decided to dedicate time for such a memorial session and to the members of the International Advisory Committee for their unanimous support. Many thanks to Oeschler's family for the heart-warming presence at the Conference and the pleasant moments, dinners and memories that were shared. We would also like to apologise to the numerous colleagues and friends who contacted us while preparing the memorial session, providing many wonderful stories about Helmut that we did not have the time to present.

\section{References}

[1] F. Saint-Laurent et al., Nucl. Phys. A 422, 307 (1984). doi:10.1016/0375-9474(84)90519-0

[2] G. Klotz-Engmann et al., Nucl. Phys. A 499, 392 (1989). doi:10.1016/0375-9474(89)90054-7

[3] M. Conjeaud et al., Phys. Lett. 159B, 244 (1985). doi:10.1016/0370-2693(85)90243-6

[4] V. Lips et al., Phys. Rev. Lett. 72, 1604 (1994). doi:10.1103/PhysRevLett.72.1604

[5] J. Cleymans et al., Phys. Rev. C 73, 034905 (2006). doi:10.1103/PhysRevC.73.034905

[6] B. Alessandro et al., J. Phys. G 321295 (2006). doi:10.1088/0954-3899/32/10/001

[7] K. Aamodt et al. (ALICE Collaboration), Eur. Phys. J. C71 1655 (2011) doi:10.1140/epjc/s10052011-1655-9 